\title{
Multiresonant Spinor Dynamics in a Bose-Einstein Condensate
}

\author{
C. Klempt, ${ }^{1}$ O. Topic, ${ }^{1}$ G. Gebreyesus,${ }^{2}$ M. Scherer, ${ }^{1}$ T. Henninger, ${ }^{1}$ P. Hyllus, ${ }^{2}$ W. Ertmer, ${ }^{1}$ L. Santos, ${ }^{2}$ and J. J. Arlt ${ }^{1}$ \\ ${ }_{1}^{1}$ Institut für Quantenoptik, Leibniz Universität Hannover, Welfengarten 1, D-30167 Hannover, Germany \\ ${ }^{2}$ Institut für Theoretische Physik, Leibniz Universität Hannover, Appelstraße 2, D-30167 Hannover, Germany
}

(Received 2 April 2009; revised manuscript received 19 October 2009; published 6 November 2009)

We analyze the spinor dynamics of a ${ }^{87} \mathrm{Rb} F=2$ condensate initially prepared in the $m_{F}=0$ Zeeman sublevel. We show that this dynamics, characterized by the creation of correlated atomic pairs in $m_{F}=$ \pm 1 , presents an intriguing multiresonant magnetic-field dependence induced by the trap inhomogeneity. This dependence is directly linked to the most unstable Bogoliubov spin excitations of the initial $m_{F}=0$ condensate, showing that, in general, even a qualitative understanding of the pair-creation efficiency in a spinor condensate requires a careful consideration of the confinement.

DOI: 10.1103/PhysRevLett.103.195302

Spinor Bose-Einstein condensates (BECs), consisting of atoms with nonzero spin, constitute an ideal scenario to investigate the interplay between internal and external degrees of freedom in a multicomponent superfluid. The competition between spin-dependent collisional interactions, the Zeeman effect, and inhomogeneous trapping results in an exciting range of fundamental phenomena. As a result, spinor BECs have attracted a lot of attention since the pioneering experiments in optical traps [1] concerning their ground state properties [2-5] and the spinor dynamics induced by the spin-changing collisions, which allow for a coherent transfer between different spin components [6,7].

Spinor BECs also provide exciting perspectives as novel sources of nonclassical states of matter. In this sense, condensates initially prepared in the $m_{F}=0$ Zeeman sublevel are especially fascinating [6-9]. In that case, the creation of correlated pairs results in the growth of macroscopic populations in $m_{F}= \pm 1$. This amplification process is ideally triggered by quantum spin fluctuations [10]. Interestingly, it closely resembles parametric amplification in optical parametric down-conversion [11], opening exciting new routes for matter-wave squeezing and atomic Einstein-Podolsky-Rosen entanglement experiments $[12,13]$.

Correlated pair creation, and in general any spinor dynamics, demands a significant rate of spin-changing collisions. In typical experiments these collisions are suppressed by the quadratic Zeeman effect (QZE) already in the presence of moderate magnetic fields [6]. However, the influence of the QZE at low fields is far from trivial $[8,14$ 17]. In particular, spin-mixing can reach a pronounced maximum for low but finite fields. This resonance, contrary to those discussed below, has a nonlinear character and has been explained in terms of phase matching [16].

The understanding of the magnetic-field dependence of the pair-creation efficiency is hence crucial for the characterization of novel spinor-based sources of nonclassical matter waves. In this Letter we show that this dependence generally presents an intriguing nonmonotonic character
PACS numbers: 67.85.Fg, 67.85.De, 67.85.Hj, 75.50.Mm

which is crucially determined by the trap inhomogeneity and cannot be explained from the physics of homogeneous BECs [18]. The pair-creation efficiency is directly linked to the instability rate, which characterizes the exponential growth of the most unstable spin excitations of the initial BEC in $m_{F}=0$. This instability rate presents pronounced maxima and minima as a function of the applied field, which result in the striking multiresonant magnetic-field dependence of the pair-creation efficiency (from $m_{F}=0$ to $m_{F}= \pm 1$ ) observed in our experiments. Along with these resonances we observe characteristic magnetization patterns which depend on both the magnetic field $[9,18,19]$ and the external confinement $[19,20]$.

To investigate this magnetic-field dependence experimentally, we initially prepare a sample of $10^{6}$ ${ }^{87} \mathrm{Rb}$-atoms in the $F=2, m_{F}=2$ state in a crossed beam optical dipole trap at a wavelength of $1064 \mathrm{~nm}$ (see Refs. [21,22] for a more detailed description). In the fol-

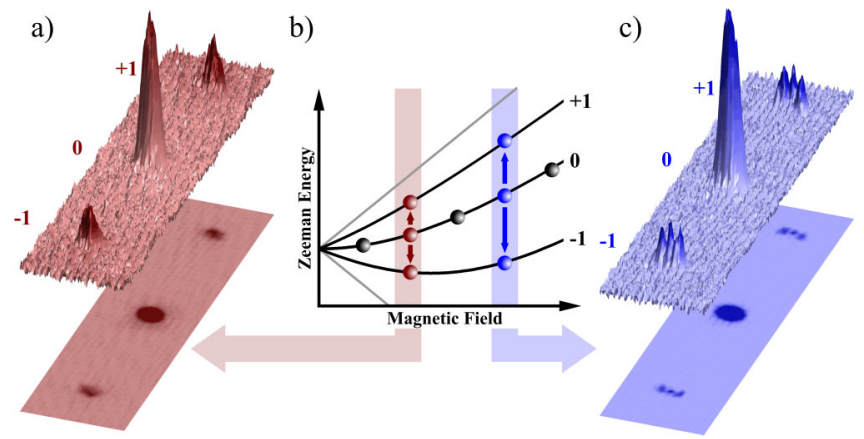

FIG. 1 (color online). Illustration of the resonances in the spinor dynamics of a Bose-Einstein condensate initially in the $\left|m_{F}=0\right\rangle$ state. For certain magnetic fields, the production efficiency of atoms in the $\left|m_{F}= \pm 1\right\rangle$ states is resonantly enhanced due to the excess energy released by the quadratic Zeeman effect. The column densities of all three Zeeman components in the plane of strongest confinement are shown in (a) and (c) in both a 3D and a density plot. Note that condensates in the $\left|m_{F}= \pm 1\right\rangle$ states are created in characteristic spatial modes depending on the particular resonance. 
lowing, the $m_{F}=X$ states are denoted by $|X\rangle$. The spin polarization of the atoms is maintained by a small guiding magnetic field. The trap depth is lowered in multiple steps to evaporatively cool the atoms from a temperature of $2 \mu \mathrm{K}$ to $90 \mathrm{nK}$, resulting in a final trap depth of $150 \mathrm{nK}$ and trapping frequencies of $(176,132,46) \mathrm{Hz}$. Typically a BEC of $7 \times 10^{4}$ atoms with a thermal fraction below $40 \%$ is formed. Subsequently, the magnetic field is raised to $78 \mathrm{G}$ and an adiabatic radio frequency passage is used to transfer the atoms to the $|0\rangle$ state within $5 \mathrm{~ms}$. At this stage, the population of other spin states is below the detection limit of $\approx 500$ atoms. After the transfer, the field is lowered to $7.9 \mathrm{G}$ within $15 \mathrm{~ms}$. During this ramp, a strong gradient of $\approx 50 \mathrm{G} / \mathrm{cm}$ is applied to expel residual atoms in the $m_{F} \neq 0$ components from the trap. Subsequent to this purification, the field is quickly lowered to a specific value between 0.12 and $2 \mathrm{G}$ within $3 \mathrm{~ms}$. This magnetic-field strength is calibrated by microwave hyperfine transitions and has a width of $4 \mathrm{mG}$ due to current noise, current drift and residual gradients. Magnetic-field gradients are below $0.2 \mathrm{mG} / \mathrm{cm}$ in all directions. The BEC is held at the chosen magnetic field for an adjustable time to allow for spinchanging collisions. Finally, the dipole trap is switched off to let the atomic cloud expand freely. During time-offlight, a strong magnetic-field gradient is applied in the vertical direction to separate the $m_{F}$ components. The number of atoms in all five $m_{F}$ components is measured by standard absorption imaging along the weak trap axis. A bimodal fit to the measured density profile of the $m_{F}=0$ component yields the condensed fraction and the temperature of the cloud. Typical density profiles after time-offlight are shown in Fig. 1.

To evaluate the onset of the spinor dynamics, we restrict our investigation to short spin evolution times and small populations in the $| \pm 1\rangle$ states. Figure 2(a) shows the magnetic-field dependence of the fraction of atoms transferred to the $| \pm 1\rangle$ states after an evolution time of $21 \mathrm{~ms}$. The magnetic-field strength $B$ is represented by the quadratic Zeeman energy $q \propto B^{2}$ which is the relevant energy scale. As expected [7], no atoms in the $| \pm 2\rangle$ states were detected at this time scale. The pair-creation efficiency shows a striking multiresonant $q$ dependence. We show below that this dependence is directly linked to the interplay between Zeeman energy, spin-changing collisions and the inhomogeneous confinement of the ensemble.

The onset of the spinor dynamics is best understood by considering the spin excitation modes of the initial condensate [18]. Depending on the quadratic Zeeman energy $q$ and the atomic interactions, these modes may become dynamically unstable, triggering an exponential population growth in $m_{F} \neq 0$ states, hence initiating the spinor dynamics. An analysis based on spin excitation modes allows for a good understanding of recent experiments in $F=1{ }^{87} \mathrm{Rb}$ condensates, including spin-texture formation after a quench $[20,23]$, and - in combination with dipolar interaction - the instability of externally induced helices [24-26]. The initial state may be represented by a spinor wave function $\boldsymbol{\Psi}_{0}(\vec{r})=\left(\psi_{-2}, \psi_{-1}, \psi_{0}, \psi_{1}, \psi_{2}\right)^{T}=$ $\left(0,0, n_{0}(\vec{r})^{1 / 2}, 0,0\right)^{T}$. The onset of the spinor dynamics (linear regime) is described by an operator $\hat{\mathbf{\Psi}}(\vec{r}, t)=$ $\left(\boldsymbol{\Psi}_{0}(\vec{r})+\delta \hat{\boldsymbol{\Psi}}(\vec{r}, t)\right) e^{-i \mu t}$, where $\mu$ is the chemical potential, $\delta \hat{\mathbf{\Psi}}(\vec{r}, t)=\left(\delta \hat{\psi}_{-2}, \delta \hat{\psi}_{-1}, \delta \hat{\psi}_{0}, \delta \hat{\psi}_{1}, \delta \hat{\psi}_{2}\right)^{T}$ describes the spin fluctuations, and the density fulfils $n_{0}(\vec{r}) \gg\left\langle\delta \hat{\psi}_{m_{F}}^{\dagger}(\vec{r}) \delta \hat{\psi}_{m_{F}}(\vec{r})\right\rangle$. Up to second order in $\delta \hat{\psi}_{m_{F}}$ we obtain a Hamiltonian for the pair creation in $| \pm 1\rangle$ [18]:

$$
\begin{aligned}
\hat{H}= & \int d^{3} \vec{r}\left\{\sum_{m_{F}= \pm 1} \delta \hat{\psi}_{m_{F}}^{\dagger}\left[\hat{H}_{\mathrm{eff}}+q\right] \delta \hat{\psi}_{m_{F}}\right. \\
& \left.+\Omega_{\mathrm{eff}}(\vec{r})\left[\delta \hat{\psi}_{1}^{\dagger} \delta \hat{\psi}_{-1}^{\dagger}+\delta \hat{\psi}_{1} \delta \hat{\psi}_{-1}\right]\right\} .
\end{aligned}
$$

Note that we may neglect the transfer into $| \pm 2\rangle$ during the first stages of the dynamics due to the much lower transfer rate from $|0\rangle$. Hence the Hamiltonian is valid for the description of the initial pair creation for BECs in both $F=1$ and $F=2$. The effective Hamiltonian for the $| \pm 1\rangle$ components is $\hat{H}_{\text {eff }}=-\hbar^{2} \nabla^{2} / 2 m+V_{\text {eff }}(\vec{r})$, with $V_{\text {eff }}(\vec{r}) \equiv V(\vec{r})+\left(U_{0}+U_{1}\right) n_{0}(\vec{r})-\mu$, where $m$ is the atomic mass, and $V(\vec{r})$ is the confining potential. The coupling coefficient $\Omega_{\text {eff }}(\vec{r})=U_{1} n_{0}(\vec{r})$ characterizes the pair creation induced by spin-changing collisions. The interaction strengths are $U_{0}=\left(7 g_{0}+10 g_{2}+18 g_{4}\right) / 35$, $U_{1}=\left(-7 g_{0}-5 g_{2}+12 g_{4}\right) / 35$ for $F=2$ and

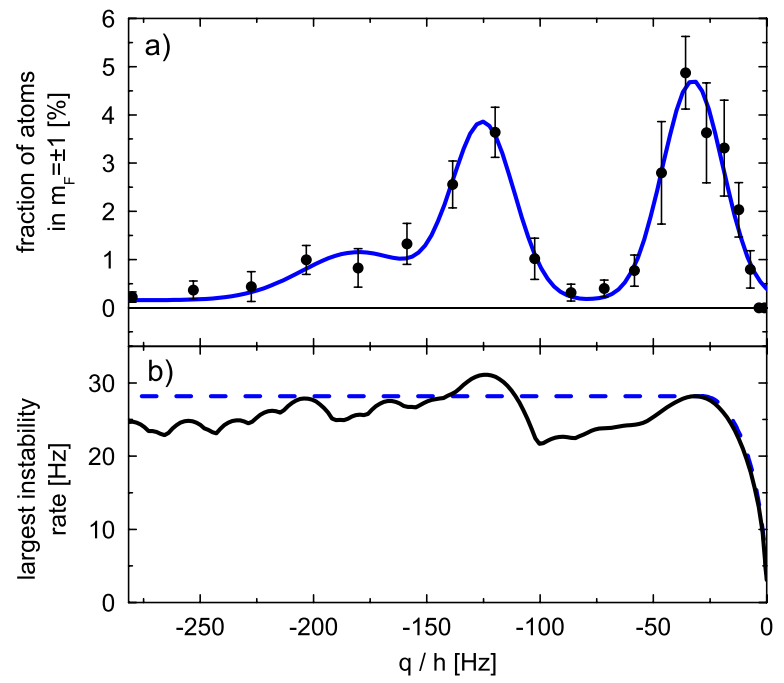

FIG. 2 (color online). (a) Fraction of atoms transferred into $\left|m_{F}= \pm 1\right\rangle$ within $21 \mathrm{~ms}$ as a function of the quadratic Zeeman energy $q$. To obtain this fraction, the number of transferred atoms per state was divided by the sum of transferred $\left|m_{F}= \pm 1\right\rangle$ and condensed $\left|m_{F}=0\right\rangle$ atoms. Because of strong shot-to-shot fluctuations 15 independent realizations were averaged at each magnetic field. The error bars indicate statistical uncertainties. The blue line is a triple Gaussian fit to guide the eye. (b) Instability rate, given by the imaginary part of the most unstable spin Bogoliubov mode, corresponding to the paircreation efficiency into $\left|m_{F}= \pm 1\right\rangle$ (solid line). The maximal instability rate for an effective homogeneous case (dashed line) lacks any resonant features. 
$U_{0}=\left(g_{0}+2 g_{2}\right) / 3, U_{1}=\left(g_{2}-g_{0}\right) / 3$ for $F=1$ condensates. They are related to $g_{F}=4 \pi \hbar^{2} a_{F} / m$, where $a_{F}$ is the $s$-wave scattering length for the collisional channel with total spin $F$ (only even due to symmetry) [2]. Note that for ${ }^{87} \mathrm{Rb} U_{1}>0$ for $F=2$ whereas $U_{1}<0$ for $F=1$.

For homogeneous BECs, $V_{\text {eff }}$ and $\Omega_{\text {eff }}$ are constants, and the spin excitations are plane waves with wave vector $\vec{k}$ and energy $\xi_{k}(q)$ obtained after diagonalizing $\hat{H}$. If the imaginary part $\operatorname{Im}\left(\xi_{k}(q)\right)$ is positive for some $k$, the BEC in $|0\rangle$ is dynamically unstable and pair production into the $| \pm 1\rangle$ state occurs. By adapting the result of Ref. [18] to the general case of spinor BECs in $F=2$ and $F=1$, we obtain three different regimes, which can be classified according to the value of $q$. BECs in the $|0\rangle$ state are (i) stable for $q>q_{\mathrm{cr}}+\left|q_{\mathrm{cr}}\right|$ (where $q_{\mathrm{cr}}=-U_{1} n_{0}$ ), (ii) unstable for $q_{\mathrm{cr}}<q<q_{\mathrm{cr}}+\left|q_{\mathrm{cr}}\right|$, where the instability rate of the most unstable mode (with $k_{\max }=0$ ) is $\Lambda(q)=$ $\sqrt{q_{\mathrm{cr}}^{2}-\left(q-q_{\mathrm{cr}}\right)^{2}} / h$; and (iii) unstable for $q<q_{\mathrm{cr}}$ with a constant instability rate $\Lambda(q)=\left|q_{\mathrm{cr}}\right| / h$ for the most unstable modes $\hbar^{2} k_{\max }^{2} / 2 m=q_{\mathrm{cr}}-q$. The $q$ dependence of $\Lambda(q)$ yields the magnetic-field dependence of the paircreation efficiency.

Although the homogeneous picture offers important insights, the observed $q$-dependence in trapped BECs is strikingly different, showing that the confinement must be considered to obtain even a qualitative understanding. This may be partially understood by comparison with the simple case of a $F=2 \mathrm{BEC}$ in a box potential $V(\vec{r})=0$ for $r<R$, and $V(\vec{r})=\infty$ otherwise. In the Thomas-Fermi regime the density $n_{0}$ of the BEC in the $|0\rangle$ state is approximately constant for $r<R$, and the energies of the Bogoliubov modes are $\xi_{n}^{2}(q)=\left(\epsilon_{n}+q\right)\left(\epsilon_{n}+q+\right.$ $\left.2 U_{1} n_{0}\right)$, with $\epsilon_{n}$ the energy of the $n$th box state. Regimes (i) and (ii) are similar to the homogeneous case. However, for regime (iii), occurring for $\eta(q) \equiv|q|-U_{1} n_{0}>\left(\epsilon_{0}+\right.$ $\left.\epsilon_{1}\right) / 2$, the most unstable mode is given by the level $\bar{n}$ whose energy $\epsilon_{\bar{n}}$ is closest to $\eta(q)$. Contrary to the infinite system $\Lambda(q)=\left|\xi_{\bar{n}}(q)\right| / h$ shows pronounced maxima and minima, reaching its maximum $U_{1} n_{0}$ only when $\eta(q)$ is resonant with a box state.

For the actual experimental conditions not only the finite confinement but also the inhomogeneity of the potential and the related inhomogeneous Thomas-Fermi density profile $n_{0}(\vec{r})$ contribute to the mode instability. This is best understood by introducing the eigenfunctions of the effective Hamiltonian $H_{\text {eff }} \phi_{n}(\vec{r})=\epsilon_{n} \phi_{n}(\vec{r})$. Note that the modes $\phi_{n}$ play a similar role to the box states in the simplified discussion above. After projecting $\delta \hat{\psi}_{m_{F}}(\vec{r})=$ $\sum_{n} \phi_{n}(\vec{r}) \hat{a}_{n, m_{F}}$, we may rewrite the Hamiltonian (1) in the form

$$
\begin{aligned}
\hat{H}= & \sum_{n, m_{F}= \pm 1}\left(\epsilon_{n}+q\right) \hat{a}_{n, m_{F}}^{\dagger} \hat{a}_{n, m_{F}} \\
& +\sum_{n, n^{\prime}} A_{n, n^{\prime}}\left(\hat{a}_{n, 1}^{\dagger} \hat{a}_{n^{\prime},-1}^{\dagger}+\hat{a}_{n^{\prime},-1} \hat{a}_{n, 1}\right),
\end{aligned}
$$

where $A_{n, n^{\prime}}=\int d^{3} \vec{r} \Omega_{\text {eff }}(\vec{r}) \phi_{n}(\vec{r})^{*} \phi_{n^{\prime}}(\vec{r})$. Note that the in- homogeneity of $V(\vec{r})$ and $n_{0}(\vec{r})$ plays a double role. First, $V_{\text {eff }}(\vec{r})$ presents a nontrivial Mexican-hat-like form (for $\left.U_{1}>0\right)$. Second, contrary to the simplified box potential, there is a significant coupling $\left(A_{n, n^{\prime}}\right)$ between different effective trap levels, induced by the inhomogeneity of $\Omega_{\mathrm{eff}}(\vec{r})$.

After diagonalizing $\hat{H}$ we obtain the spin Bogoliubov modes $\xi_{\nu}(q)$. Figure 2(b) shows the magnetic-field dependence of the maximal instability rate $\Lambda(q)=$ $\max _{\nu}\left|\operatorname{Im}\left(\xi_{\nu}(q)\right)\right| / h$ for the experimental parameters of Fig. 2(a). In the unstable regime with low $|q|$ we may approximate the maximal instability rate by $\Lambda(q) \simeq$ $\sqrt{\tilde{q}_{\mathrm{cr}}^{2}-\left(q-\tilde{q}_{\mathrm{cr}}\right)^{2}} / h$ with an effective $\tilde{q}_{\mathrm{cr}}(\simeq-30 \mathrm{~Hz}$ in Fig. 2). However, as expected from the discussion above, this growth is not followed by a constant instability rate for larger $|q|$. On the contrary, $\Lambda(q)$ shows pronounced maxima and minima, which lead to a strongly enhanced or reduced pair-creation efficiency into $| \pm 1\rangle$ due to the exponential nature of the growth. Note that the position of the maxima and minima of the observed pair-creation efficiency in our experiments is in excellent agreement with the position of the calculated maxima and minima of the instability rate in Fig. 2. However, the evaluation of the resonance strength is more subtle. The experimentally observed resonance at low $|q|$ seems to be stronger than the one at high $|q|$, whereas the calculated instability rate is slightly smaller for the resonance at low $|q|$. This is not a disagreement, since the absolute population is not only determined by the growth rate but also by the initial condition. In fact, the resonant growth can be initiated by spuriously produced atoms in $| \pm 1\rangle$ and by vacuum spin fluctuations. Since these triggering mechanisms contribute differently for the two resonances, they can fully explain the experimental results [10].

The agreement in the resonance position is maintained for different experimental parameters. In particular, we vary the atom number in our experiments by introducing an additional hold time of up to $30 \mathrm{~ms}$ before the purification step, which allows for a controlled loss of atoms. By varying this hold time, the total atom number $N$ is changed without changing any of the other experimental parameters. Figure 3 shows the position of the resonance peak at

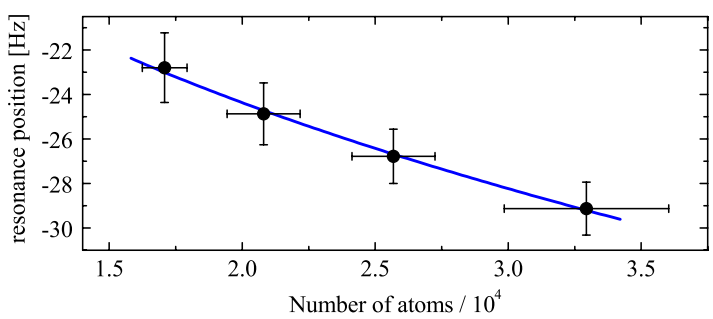

FIG. 3 (color online). Position of the lowest energy spinor dynamics resonance, recorded for various atom numbers $N$ of the initial condensate in the $\left|m_{F}=0\right\rangle$ state. The blue line represents a fit to a power law $N^{\gamma}$, yielding an exponent of $\gamma=$ $0.36 \pm 0.02$. 


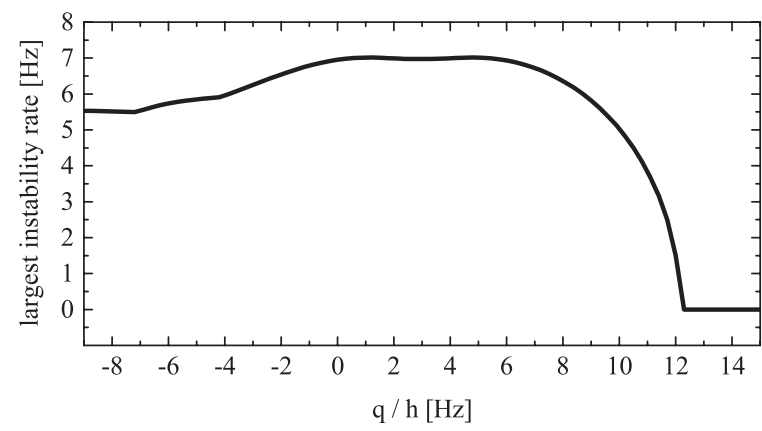

FIG. 4. Instability rate as a function of the quadratic Zeeman energy for the parameters of Ref. [9], $\left(\omega_{x}, \omega_{y}, \omega_{z}\right)=$ $2 \pi \times(440,39,4.2) \mathrm{Hz}$ and $N=2 \times 10^{6}$ atoms. A constant maximal instability rate for $0<q<6 \mathrm{~Hz}$ is followed by a significant decay of the conversion efficiency for $q<0$.

low $|q|$ (obtained from a Gaussian fit) for different numbers of atoms. The resonant $q$ value increases with the number of atoms according to $N^{0.36 \pm 0.02}$. This exponent of $\approx 2 / 5$ closely resembles the dependence of the peak density on the number of atoms in the $|0\rangle$ state in the Thomas-Fermi regime. Hence the position of the resonance at low $|q|$ depends linearly on the BEC density, as expected from the linear scaling of $V_{\text {eff }}$ and $\Omega_{\text {eff }}$ with the density.

Our results show that the pair-creation efficiency directly reflects the instability of the spin Bogoliubov modes. This picture is general and may be applied to other experiments on spinor BECs. In particular, Fig. 4 shows the instability rate for the case of recent experiments with ${ }^{87} \mathrm{Rb}$ in $F=1$ [9]. Our theory predicts a single resonance with a constant instability rate for $0<q<6 \mathrm{~Hz}$, and a clear decay of the pair-creation efficiency for $q<0$ (which was unexpected from the simplified homogeneous approximation), in excellent agreement with the reported results [9]. We stress that the single-resonant character (as in Ref. [9]) or multiresonant character (as in our case) of the dynamics largely depends on atomic density and external confinement.

In conclusion, the pair-creation efficiency in a spinor BEC initially in the $|0\rangle$ state is characterized by a multiresonant magnetic-field dependence, which results from the nontrivial interplay between QZE, spin-changing collisions and the finite size and inhomogeneity of both the trapping potential and the density of the BEC in the $|0\rangle$ state. We have shown that this inhomogeneity is crucial even for the qualitative understanding of the spinor dynamics. In particular, the trap-dependent modulation of the most unstable spin Bogoliubov mode is directly reflected by a strong enhancement or suppression of the spinor dynamics for particular magnetic-field values. On the resonances, we observe the spontaneous emergence of characteristic magnetization patterns [27]. It is also important to stress that, in particular, for Rb BECs in $F=1$, the magnetic dipole-dipole interaction may play a significant role not only in the spatial magnetization pattern $[19,24,28]$ but also in the pair production efficiency.
The characterization of the pair-creation efficiency constitutes a comprehensive description of a parametric amplifier for matter waves. The next challenge is the evaluation of the amplifier input which can be of classical or quantum nature. A realization where the presented parametric amplifier acts on pure vacuum spin fluctuations constitutes a promising source of nonclassical states of matter [10]. This will open exciting perspectives for the detailed analysis of matter-wave entanglement and squeezing during the parametric amplification of the $| \pm 1\rangle$ pairs $[12,13]$, possibly allowing for the production of atomic Einstein-Podolsky-Rosen pairs [12].

We acknowledge support from the Centre for Quantum Engineering and Space-Time Research QUEST, from the Deutsche Forschungsgemeinschaft (SFB 407), and the European Science Foundation (EuroQUASAR).

[1] J. Stenger et al., Nature (London) 396, 345 (1998).

[2] T.-L. Ho, Phys. Rev. Lett. 81, 742 (1998).

[3] T. Ohmi and K. Machida, J. Phys. Soc. Jpn. 67, 1822 (1998).

[4] M. Koashi and M. Ueda, Phys. Rev. Lett. 84, 1066 (2000).

[5] C. V. Ciobanu, S.-K. Yip, and T.-L. Ho, Phys. Rev. A 61, 033607 (2000).

[6] M.-S. Chang et al., Phys. Rev. Lett. 92, 140403 (2004).

[7] H. Schmaljohann et al., Phys. Rev. Lett. 92, 040402 (2004).

[8] T. Kuwamoto, K. Araki, T. Eno, and T. Hirano, Phys. Rev. A 69, 063604 (2004).

[9] S. R. Leslie et al., Phys. Rev. A 79, 043631 (2009).

[10] C. Klempt et al. (to be published).

[11] P. Meystre and M. Sargent, Elements of Quantum Optics (Springer, Berlin, 2007), 4th ed.

[12] H. Pu and P. Meystre, Phys. Rev. Lett. 85, 3987 (2000).

[13] L.-M. Duan, A. Sørensen, J. I. Cirac, and P. Zoller, Phys. Rev. Lett. 85, 3991 (2000).

[14] J. Kronjäger et al., Phys. Rev. A 72, 063619 (2005).

[15] W. Zhang et al., Phys. Rev. A 72, 013602 (2005).

[16] J. Kronjäger et al., Phys. Rev. Lett. 97, 110404 (2006).

[17] A. T. Black et al., Phys. Rev. Lett. 99, 070403 (2007).

[18] A. Lamacraft, Phys. Rev. Lett. 98, 160404 (2007).

[19] J.D. Sau, S. R. Leslie, D. M. Stamper-Kurn, and M. L. Cohen, Phys. Rev. A 80, 023622 (2009).

[20] M. Baraban, H. F. Song, S. M. Girvin, and L. I. Glazman, Phys. Rev. A 78, 033609 (2008).

[21] C. Klempt et al., Phys. Rev. A 78, 061602(R) (2008).

[22] C. Klempt et al., Eur. Phys. J. D 48, 121 (2008).

[23] L. E. Sadler et al., Nature (London) 443, 312 (2006).

[24] M. Vengalattore, S. R. Leslie, J. Guzman, and D. M. Stamper-Kurn, Phys. Rev. Lett. 100, 170403 (2008).

[25] R.W. Cherng, V. Gritsev, D. M. Stamper-Kurn, and E. Demler, Phys. Rev. Lett. 100, 180404 (2008).

[26] R. W. Cherng and E. Demler, arXiv:0806.1991v1.

[27] Structure and symmetry of the patterns are described by the presented framework and will be published elsewhere.

[28] T. Świsłocki, M. Brewczyk, M. Gajda, and K. Rzkażewski, arXiv:0901.1763. 